\title{
Diagnostic and predictive significance of serum microRNA-7 in esophageal squamous cell carcinoma
}

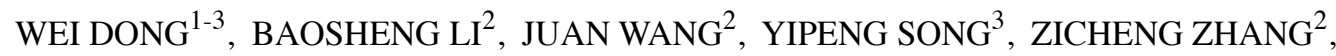 \\ CHENGRUI FU ${ }^{2}$ and PEILIANG ZHANG ${ }^{4}$
}

\author{
${ }^{1}$ School of Medicine, Shandong University, Jinan, Shandong 250012; ${ }^{2}$ Department of Radiation Oncology, \\ Shandong Cancer Hospital, Jinan, Shandong 250117; ${ }^{3}$ Department of Radiation Oncology, Yuhuangding Hospital, \\ Yantai, Shandong 264000; ${ }^{4}$ Department of Radiation Oncology, Yishui Central Hospital, \\ Yishui, Shandong 276400, P.R. China
}

Received October 3, 2015; Accepted November 5, 2015

DOI: $10.3892 / o r .2015 .4499$

\begin{abstract}
MicroRNA-7 has been reported to participate in tumorigenesis and progression by several signaling pathways in various tumors. However, its potential as a serum diagnostic factor and predictive biomarker for esophageal squamous cell carcinoma (ESCC) has not been studied. Serum samples were collected from 105 pathologically proven ESCC patients and 30 age- and gender-matched healthy controls. All patients were treated with concurrent chemoradiotherapy (CRT). Real-time polymerase chain reaction was carried out to measure the serum miR-7 expression level. The data were compared among radio-sensitive and radio-resistant groups, and healthy volunteers to elucidate the diagnostic and predictive value of miR-7 expression. Finally, in vitro experiments are used to clarify the mechanisms of the miR-7. In the present study, we found that the serum miR-7 level of ESCC patients was 4.74-fold lower as compared with healthy subjects, indicating that serum miR-7 expression could be an excellent diagnostic factor. The serum miR-7 expression level for these responsive patients was 2.34-fold higher than that for non-responsive patients, indicating it as a valuable biomarker for predicting treatment response of ESCC patients to concurrent chemoradiation treatment. We also found that miR-7 levels are strongly correlated with tumor length and the status of lymph node metastasis $(\mathrm{P}<0.05)$. In contrast, the responsiveness of therapy is significantly correlated with CEA $(\mathrm{P}<0.05)$, Cyfra21-1 $(\mathrm{P}<0.05)$, serum miR-7 level $(\mathrm{P}<0.05)$ and myelosuppression $(\mathrm{P}<0.01)$. In addition, the experimental data also suggest that miR-7 can interfere with EGFR mRNA translation. In ESCC patients, serum miR-7 has the potential to serve as a noninvasive
\end{abstract}

Correspondence to: Dr Baosheng Li, Department of Radiation Oncology, Shandong Cancer Hospital, 440 Jiyan Road, Jinan, Shandong 250117, P.R. China

E-mail: baoshli1963@163.com

Key words: miR-7 expression, esophageal squamous cell carcinoma, chemoradiation therapy, radiosensitivity, EGFR biomarker of diagnosis and predicting treatment responses to concurrent chemoradiation therapy. ESCC patients with lower Cyfra21-1 and CEA, higher miR-7 and severe myelosuppression were much more sensitive to CRT. In addition, miR-7 may function by interfering with EGFR mRNA translation, but not degradation.

\section{Introduction}

Esophageal carcinoma is the sixth most common cause of cancer-related death worldwide. China is one of the highincidence countries and esophageal cancer accounts for approximately 150,000 death each year, nearly a quarter of all cancer deaths (1) in the country. Esophageal cancer is usually diagnosed at an advanced stage, making curative surgical resection, which is initially recommend for early stage cases, feasible for only $30-40 \%$ of patients (2). The outcome of surgery for patients with such an aggressive tumor is still unsatisfactory, with a 5-year survival rate less than $20 \%$ (3). Accumulating evidence from randomized clinical trials supports the use of neoadjuvant chemoradiotherapy (CRT), which is shown to improve resectability and survival in patients with locally advanced esophageal cancer, although mixed results have been reported (4). The variations in clinical responses to CRT are most evident for esophageal squamous cell carcinoma (ESCC), and the survival rates between responders and non-responders are quite different even with the same clinical stage $(5,6)$. Therefore, there is a compelling need to identify novel biomarkers that hold promise of precisely predicting tumor response to CRT to tailor treatments for different ESCC patients and enhance survival (7).

MicroRNAs (miRNAs) are endogenous, short, non-coding RNAs that can regulate the expression of target genes by binding to RNA-binding proteins to control the occurrence and development of the disease including cancer. It was first characterized in 1993, since then, more and more studies have focused on the role of miRNA-small molecules in the cellular processes and pathways including the differentiation, progression, apoptosis and proliferation of different disease. In 2002, Calin et al (8) first linked miRNAs with cancer progression. Since then, increased number of studies have reported the 
role of miRNAs in carcinogenesis, indicating that miRNAs are closely related to the process of epithelial-mesenchymal transition $(9,10)$, characteristic of cancer stem cells $(10,11)$, the initiation of tumor invasion and metastasis $(12,13)$, and the therapeutic response to chemotherapy or radiotherapy (14). There is growing evidence to indicate miRNAs as biomarkers, expression profile of miRNAs are different in cancer and normal tissues, even in different organs or tissues $(15,16)$. Recent studies have reported that tissue-specific miRNAs are consistently detected in circulating samples, and cancer tissue-specific miRNAs also have been found in the circulation at different stages of the disease (17). In addition, more and more reports have demonstrated that tumor cells release miRNAs into the circulation and these circulating miRNAs are in a remarkably stable, cell-independent form which is protected from endogenous RNase activity in the bloodstream, suggesting potential opportunities for using circulating miRNAs as blood-based, non-invasive biomarkers for molecular diagnostics, physiological and pathological status, including cancer (18-21).

MicroRNA-7 (miR-7) is an intronic miRNA that resides in the first intron of the heterogeneous ribonuclear protein $\mathrm{K}$ gene on chromosome 9, and is evolutionarily conserved across all species. Previous findings suggested that miR-7 participates in tumorigenesis and progression by several signaling pathways in various types of tumors (22-27). Li and Carthew (28) have certified that 3'-untraslated regions of human EGFR contains miR-7 complementary sites, which enable it to act on EGFR expression. Furthermore, resent studies have reported that miRNA-7 can affect sensitivity to chemotherapy by MRP1 (29) and radiotherapy by EGFR (30), providing opportunities for the development of miRNA-based therapies and/or biomarkers in CRT of cancer patients.

To investigate the functional roles of miR-7 in radioresponse of ESCC and its underlying mechanism, we detected the serum miR-7 expression in ESCC and analyzed its association with clinical response of ESCC to CRT and evaluated the possibility of using serum miR-7 as a diagnostic and predicting factor for ESCC. Furthermore, we investigated the potential miR-7 role in transfected cellular model of ECA-109, identifying EGFR as a direct downstream target of miR-7.

\section{Materials and methods}

Ethics statement. This research involved human participants, thus serum-based specimen collection and studies were approved by the Institutional Review Boards of Shandong Cancer Hospital, the Shandong Academy of Medical Sciences. All participants provided written consent and indicated willingness to donate their blood samples for research.

Samples and treatment regimen. Serum samples were collected from the peripheral venous blood of 105 patients and 30 healthy volunteers at the Department of Radiation Oncology, Shandong Cancer Hospital. Clinical data of enrolled patients including gender, age, tumor locations, status of lymph node and distant metastases, the maximum diameter of tumor and tumor differentiation were recorded. Entry criteria and treatment regimen for the present study have been described in our published study (31). Immediately after collection, the serum samples were snap-frozen in liquid nitrogen and then stored at $-80^{\circ} \mathrm{C}$ for RNA extraction for quantitative RT-PCR (qRT-PCR). Response Evaluation Criteria In Solid Tumors (RECIST), the guidelines recommended by the World Health Organization (32) was applied in the present study to evaluate the response of ESCC patients to CRT (concurrent radio-chemotherapy): each patient's response was defined as complete remission (CR), partial remission (PR), stable disease (SD) or progressive disease (PD).

Cell line and cell culture. ECA-109 cells, a well differentiated human ESCC cell line, were provided by Tianjin Cancer Hospital. Cells were maintained in RPMI-1640 medium (HyClone, USA) containing 10\% heat-inactivated fetal bovine serum (FBS; Gibco), $100 \mathrm{U} / \mathrm{ml}$ penicillin, $100 \mathrm{U} / \mathrm{ml}$ streptomycin at $37^{\circ} \mathrm{C}$ in a humidified atmosphere of $5 \% \mathrm{CO}_{2}$. Cells were passaged every 2-3 days to maintain exponential growth.

miRNA transfection. The mimic of miR-7 was purchased from GenePharma (Shanghai, China). The ECA-109 cells were transfected with $100 \mathrm{nM}$ of miR-7 mimic or their corresponding negative controls. Lipofectamine 2000 (Invitrogen, San Diego, CA, USA) was used for cell transfection. Transfection complexes were added into the culture plates and incubated for $4 \mathrm{~h}$, and then replaced by fresh medium according to the manufacturer's instructions.

Screening and verification of circulating miRNAs by RT-PCR. Total RNA from human samples, including miRNAs, was extracted from $400 \mu \mathrm{l}$ of serum using the mirVana PARIS RNA isolation kit (Ambion, Austin, TX, USA) according to the manufacturer's instructions with the cell-miR-39 spike-in (Sangon Biotech, Shanghai, China). A mirVana miRNA column (Ambion) was used to collect total RNA. The bound RNA was cleaned with the buffers provided by the manufacturer to remove impurities and eluted in a final volume of $50 \mu$. Total RNA from cells were isolated using TRIzol reagent (Invitrogen) according to the manufacturer's instructions.

To analyze the expression miR-7, quantitative real-time PCR was conducted using TaqMan fluorogenic probes. miR-7 was detected by qRT-PCR using the mirVana ${ }^{\mathrm{TM}}$ qRT-PCR primer set and the mirVana ${ }^{\mathrm{TM}}$ qRT-PCR miRNA detection kit (Applied Biosystems, San Diego, CA, USA) according to the manufacturer's instructions, which were previously described (31).

Relative levels of EGFR mRNA in cells were examined by SYBR-Green real-time quantitative reverse transcription-PCR and normalized to $\beta$-actin mRNA. Reverse transcriptions using the PrimeScript ${ }^{\circledR}$ RT Master Mix (Perfect Real-Time), and qRT-PCR was performed using SYBR ${ }^{\circledR}$ Premix Ex Taq $^{\text {TM }}$ II (Perfect Real-Time) (both from Takara Bio, Inc., Japan) according to the manufacturer's instructions.

All qRT-PCRs were performed in duplicate, and the data are presented as mean \pm standard error of the mean. Real-time polymerase chain reaction (RT-PCR) was carried out on the ABI 7900 Real-Time PCR System (Applied Biosystems). Relative microRNA expression was calculated with the $2^{-\Delta \Delta \mathrm{Ct}}$ method $(31,33)$, where $\Delta \Delta \mathrm{Ct}=(\mathrm{Ct}$ gene of interest $-\mathrm{Ct}$ normalized gene $)$ of $(\mathrm{CR}+\mathrm{PR})-(\mathrm{Ct}$ gene of interest $-\mathrm{Ct}$ normalized gene) of (SD+PD). 
Western blot analysis. Cell lysates were prepared in lysis buffer [0.15 M NaCl, $50 \mathrm{mM}$ Tris- $\mathrm{Cl}$ (pH 7.5), 2 mM EDTA, $0.5 \%$ Triton-100, $5 \mathrm{mM}$ DTT, $0.2 \mathrm{mM}$ phenylmethylsulfonyl fluoride (PMSF), $2 \mu \mathrm{g} / \mathrm{ml}$ apoptinin] following $48 \mathrm{~h}$ transfection. Protein concentrations of total cell lysates were measured by Bio-Rad protein assay, and $50 \mu \mathrm{g}$ of total cell lysates/lane was separated by $10 \%$ SDS-PAGE. After electrophoresis, the protein was transferred to polyvinylidene defluoride (PVDF) membrane, which was blocked with $5 \%$ non-fat milk in Trisbuffered saline with Tween-20 (TBST) [50 mmol/l Tris-HCl (pH 7.6), $150 \mathrm{mmol} / 1 \mathrm{NaCl}, 0.1 \%$ Tween-20] for $1.5 \mathrm{~h}$ at room temperature. Immunoblotting was performed with rabbit anti-EGFR (1:500; CST), and mouse anti- $\beta$-actin (1:500; Santa Cruz) primary antibodies after washing the membrane 3 times in TBST buffer. Membranes were subsequently probed with horseradish peroxidase-conjugated secondary antibody (1:5,000; Zhongshan Biotechnology, China), developed by chemiluminescence and exposed to X-ray film. Densitometry was performed with gel imaging system (AlphaImager 2200; Pharmacia Biotech Co., USA). All experiments were performed in triplicate.

Statistical analysis. All clinicopathological variables and circulating miRNA levels were analyzed using PASW Statistics, Windows software version 17.0 (SPSS, Inc., Chicago, IL, USA). An unpaired t-test was performed to compare the differences in serum miRNA levels between groups. Chi-square test and logistic regression analysis were used to evaluate the association between serum miR-7 relative expression and clinical pathological variables. All tests were two-sided and $\mathrm{P}<0.05$ was considered to indicate a statistically significant result.

\section{Results}

Relative levels of serum miR-7 expression. The result analyses revealed that the $\mathrm{Ct}$ of $\mathrm{miR}-7$ expression was $15.15 \pm 1.80$ [95\% confidence interval (CI)] for the radiosensitive group and $16.38 \pm 1.44(95 \% \mathrm{CI})$ for the radioresistant group, as shown in Fig. 1. Based on statistical analysis, the relative miR-7 serum level was significantly higher in the SD+PD group when compared with the $\mathrm{CR}+\mathrm{PR}$ group $(\mathrm{P}<0.05)$. The mean miR-7 serum levels differ by 2.34 -fold between these two groups of patients, indicating that miR-7 may serve as a biomarker for predicting the response of ESCC patient to CRT. Fig. 2 presents the relative expression of serum miR-7 between two different groups.

The serum miR-7 level of healthy volunteers was $13.41 \pm 1.56$ (95\% CI). As shown by the small range, compared with ESCC patients, the serum miR-7 level in healthy subjects is 3.35 times higher than that in the radiosensitive group $(\mathrm{P}<0.01)$ and 7.84 times higher than that in the radioresistant group $(\mathrm{P}<0.01)$. The comparison between the healthy subjects and ESCC patients illustrates the possibility that serum miR-7 may also hold promise as a valuable diagnostic marker. Furthermore, the ROC curve was plotted to identify a cut-off value that could distinguish ESCC from healthy control. ROC curve analysis showed that at the optimal cut-off, serum miR-7 had a $78.1 \%$ sensitivity and a $83.3 \%$ specificity in separating ESCC from normal healthy control with an AUC of 0.841 (Fig. 4).

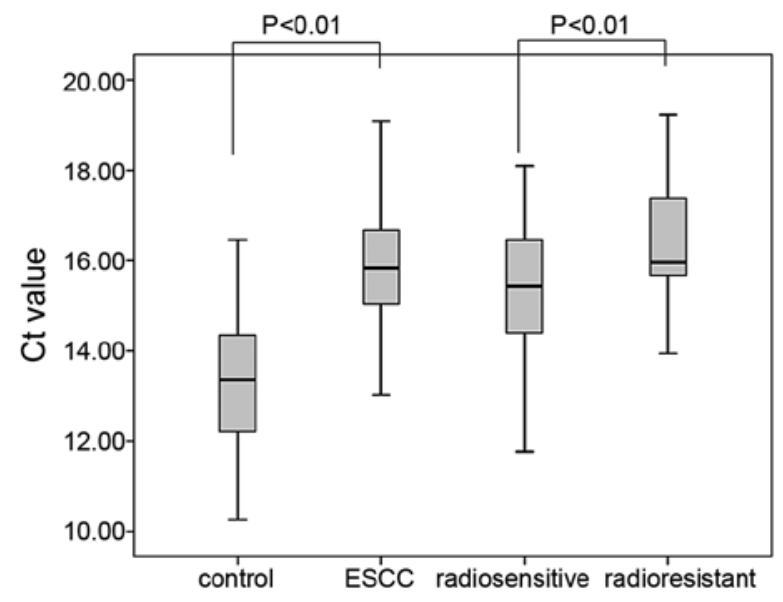

Figure 1. Ct value of serum microRNA-7 detected by real-time PCR.



Figure 2. Relative serum microRNA-7 expression: left, the serum miR-7 level in healthy subjects is 4.74 times higher than that in the ESCC patients; right, the serum miR-7 level in CRT-sensitive is 2.34 times higher than that in the CRT-resistant group ( $\left.\mathrm{P}<0.01 ;{ }^{*} \mathrm{P}<0.05\right)$.

Relationship between serum miRNA-7 expression and clinicopathological features. In addition to examining the expression of miRNAs in serum, the relationship between miR-7 expression and clinicopathological features of enrolled ESCC patients was examined. By the median value, we demarcate high and low miR-7 levels. As shown in Table I, there is no correlation between age and gender and relative miR-7 serum levels. The relative miR-7 serum level is significantly correlated with tumor length and the status of lymph node metastasis. The relative miR-7 serum level is significantly lower in patients with longer tumor compared with patients with shorter ones $(\mathrm{P}<0.05)$ and in patients with lymph node involvement compared with patients without lymph node involvement $(\mathrm{P}<0.05)$. Furthermore, the relationship between the $\mathrm{Ct}$ value of miR-7 and tumor length was examined by calculating Pearson's correlation coefficient. Our results showed a correlation $(\mathrm{r}=0.489 ; \mathrm{P}<0.01)$ between the serum miR-7 levels and the tumor length in ESCC patients (Fig. 3).

Moreover, Table I also presents the relationships between effectiveness of CRT and clinicopathological factors. As is shown in the table, the responsiveness of therapy is significantly correlated with carcinoembryonic antigen $(\mathrm{CEA})(\mathrm{P}<0.05)$, 




Figure 3. Relationship between levels of serum miR-7 and tumor length.

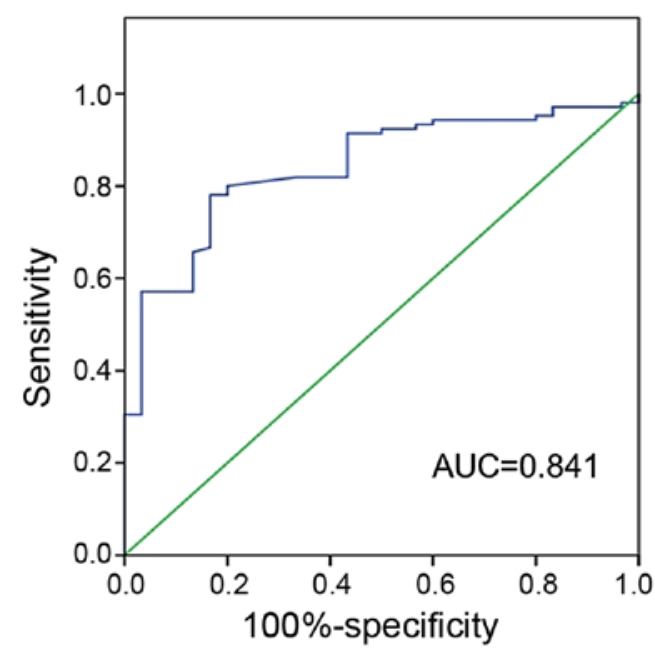

Figure 4. The ROC curve for miR-7. ROC analysis was performed to determine the sensitivity and specificity with the value of AUC.

Cyfra21-1 $(\mathrm{P}<0.05)$, serum miR-7 level $(\mathrm{P}<0.05)$ and the myelosuppression $(\mathrm{P}<0.01)$. Furthermore, by logistic regression analysis, the $\mathrm{CR}+\mathrm{PR}$ rates of $\mathrm{CRT}$ were significantly associated with the levels of Cyfra21-1 [P=0.038; overall remission $(\mathrm{OR})=0.296 ; 95 \% \mathrm{CI}$ for $\mathrm{OR}=0.094-0.934], \mathrm{CEA}(\mathrm{P}=0.022$; $\mathrm{OR}=0.276 ; 95 \% \mathrm{CI}$ for $\mathrm{OR}=0.091-0.833)$, miR-7 $(\mathrm{P}=0.019$; $\mathrm{OR}=0.265$; $95 \% \mathrm{CI}$ for $\mathrm{OR}=0.087-0.800)$, and myelosuppression $(\mathrm{P}=0.000, \mathrm{OR}=3.347,95 \% \mathrm{CI}$ for $\mathrm{OR}=1.737-6.449)$ before treatments shown in Table II. Thus, ESCC patients with lower Cyfra21-1 and CEA, higher miR-7 and severe myelosuppression were much more sensitive to CRT.

Downregulation of EGFR by miR-7. In previous studies, EGFR has been identified as an important downstream factor of miR-7 $(22,28,30)$, therefore, we focused on EGFR for further functional analyses. Compared with control subjects without any treatment, transfection of miR-7 into ECA-109 cells suppressed EGFR protein expression (Fig. 5A). However, no significant change of EGFR mRNA level was found after transfection of miR-7 mimic (Fig. 5B), suggesting that miR-7 can interfere with EGFR mRNA translation, but not degradation.

\section{Discussion}

Chemoradiotherapy (CRT) play a very important role in the treatment of esophageal cancer, however, it remains unclear on the prediction of treatment responsiveness to CRT. It is important to identify robust factors that will predict response to CRT, and will also facilitate appropriate patient selection and avoid unnecessary delays in patients at high risk of locoregional recurrence upon chemoradiation (4). Wieder et al (34) and Suzuki et al (35) have given some clues from uptake value and changes in metabolic activity in PET/CT that indicating tumor response and patient survival.

Recently, numerous studies focused on the factors that affect tumor responses to CRT, such as the presence of tumor hypoxia (36), tumor microenvironment (37), DNA damage repair (38), cell cycle checkpoint, apoptosis (39) and radio-related signal transduction pathways (40). Understanding the regulatory mechanisms of miRNA in tumor radiosensitivity from these diverse aspects has also become an intense area of interest. Although there has been great progress in the diagnosis, prediction of response to treatment and prognosis of ESCC in the past decades, identifying new biomarkers may greatly benefit the early detection screening methods.

Great advances concerning miRNA-based therapeutics have been made in various human diseases, including cancer. Recent studies have verified that dysregulation of miRNA expression in human diseases may act as diagnostic, and prognostic factors as well as predicting response to chemotherapy or/and radiotherapy biomarkers. miR-7 has previously been characterized as a tumor-suppressor miRNA in several human cancers by targeting a number of key signaling molecules $(22,25,41)$. The present study showed that serum miR-7 levels were downregulated (4.74-fold-change) in patients with ESCC compared with healthy controls, indicating that it may be a useful biomarker for early diagnosis. However, the downregulation is not unique to ESCC. For example, the level of serum miR-7 is decreased in glioblastoma, and increased miR-7 inhibits glioma cell proliferation by inhibiting the EGFR and AKT pathways (24). Downregulation of miR-7 in breast cancer has also been proven in the study by Kong et al (42), who demonstrated the inhibition of epithelial-to-mesenchymal transition and metastasis of breast cancer cells via targeting FAK expression. Previously studies have demonstrated that miR-7 is also downregulated in advanced tongue squamous cell carcinoma cell lines $(43,44)$. Therefore, our current results are consistent with previous findings.

The present study shows that the presence of ESCC is associated with a suppressed level of serum miR-7, and the degree of suppression is correlated with tumor length and lymph node status. An interesting feature of our study is the existence of a statistically significant correlation between lower serum miR-7 expression in ESCC and longer tumor, as well as positive lymph node metastasis, which are the main prognostic factors for ESCC. ROC curve analysis showed that at the optimal cut-off, serum miR-7 had a $78.1 \%$ sensitivity and a $83.3 \%$ specificity in separating ESCC from normal healthy control with an AUC of 0.841 (Fig. 3). Such findings imply that miR-7 may be involved in the initiation and progression of cancer. 
Table I. Relationships between effectiveness of chemoradiotherapy (CRT) and clinicopathological factors as well as serum levels of tumor markers.

\begin{tabular}{|c|c|c|c|c|c|c|c|c|}
\hline \multirow[b]{2}{*}{ Elements } & \multicolumn{2}{|c|}{ MicroRNA-7 } & \multirow[b]{2}{*}{$\chi^{2}$} & \multirow[b]{2}{*}{ P-value } & \multicolumn{2}{|c|}{ Effectiveness } & \multirow[b]{2}{*}{$\chi^{2}$} & \multirow[b]{2}{*}{ P-value } \\
\hline & High expression & Low expression & & & $\mathrm{CR}+\mathrm{PR}$ & $\mathrm{SD}+\mathrm{PD}$ & & \\
\hline Gender & & & 0.116 & 0.733 & & & 0.012 & 0.914 \\
\hline Male & 35 & 34 & & & 41 & 28 & & \\
\hline Female & 17 & 19 & & & 21 & 15 & & \\
\hline Age (years) & & & 0.97 & 0.325 & & & 1.155 & 0.282 \\
\hline$\geq 60$ & 38 & 34 & & & 40 & 32 & & \\
\hline$<60$ & 14 & 19 & & & 22 & 11 & & \\
\hline Tumor location & & & 0.457 & 0.796 & & & 3.545 & 0.17 \\
\hline Upper third & 16 & 14 & & & 22 & 8 & & \\
\hline Middle third & 28 & 32 & & & 32 & 28 & & \\
\hline Lower third & 8 & 7 & & & 8 & 7 & & \\
\hline Length & & & 7.505 & 0.023 & & & 1.662 & 0.436 \\
\hline$\leq 4.0$ & 33 & 20 & & & 34 & 19 & & \\
\hline $4.1-6.0$ & 13 & 19 & & & 16 & 16 & & \\
\hline$>6.0$ & 6 & 14 & & & 12 & 8 & & \\
\hline Tumor differentiation & & & 1.157 & 0.561 & & & 1.557 & 0.459 \\
\hline High & 13 & 11 & & & 13 & 11 & & \\
\hline Moderate & 28 & 26 & & & 35 & 19 & & \\
\hline Poor & 11 & 16 & & & 14 & 13 & & \\
\hline Lymph node metastasis & & & 4.197 & 0.04 & & & 0.264 & 0.607 \\
\hline Positive & 21 & 32 & & & 30 & 23 & & \\
\hline Negative & 31 & 21 & & & 32 & 20 & & \\
\hline Distant metastasis & & & 0.03 & 0.861 & & & 0.012 & 0.912 \\
\hline Positive & 12 & 13 & & & 15 & 10 & & \\
\hline Negative & 40 & 40 & & & 47 & 33 & & \\
\hline Smoking history & & & 0.234 & 0.628 & & & 0.367 & 0.545 \\
\hline Positive & 26 & 24 & & & 28 & 22 & & \\
\hline Negative & 26 & 29 & & & 34 & 21 & & \\
\hline Drinking history & & & 0.106 & 0.745 & & & 0.024 & 0.876 \\
\hline Positive & 19 & 21 & & & 24 & 16 & & \\
\hline Negative & 33 & 32 & & & 38 & 27 & & \\
\hline Family history & & & 0.003 & 0.958 & & & 0.007 & 0.935 \\
\hline Positive & 12 & 12 & & & 14 & 10 & & \\
\hline Negative & 40 & 41 & & & 48 & 33 & & \\
\hline CEA & & & 0.497 & 0.481 & & & 5.237 & 0.021 \\
\hline$\leq 3.3$ & 35 & 39 & & & 49 & 25 & & \\
\hline$>3.3$ & 17 & 14 & & & 13 & 18 & & \\
\hline Cyfra21-1 & & & 0.293 & 0.589 & & & 5.901 & 0.015 \\
\hline$\leq 3.4$ & 35 & 33 & & & 46 & 22 & & \\
\hline$>3.4$ & 17 & 20 & & & 16 & 21 & & \\
\hline Myelosuppression & & & 4.024 & 0.403 & & & 25.076 & 0.000 \\
\hline 0 & 17 & 13 & & & 7 & 23 & & \\
\hline I & 20 & 20 & & & 26 & 14 & & \\
\hline II & 10 & 15 & & & 21 & 4 & & \\
\hline III & 3 & 5 & & & 6 & 2 & & \\
\hline IV & 2 & 0 & & & 2 & 0 & & \\
\hline miR-7 level & & & & & & & 4.418 & 0.036 \\
\hline Higher & & & & & 36 & 16 & & \\
\hline Lower & & & & & 26 & 27 & & \\
\hline
\end{tabular}

$\mathrm{CR}$, complete remission; PR, partial remission; SD, stable disease; PD, progressive disease; CEA, carcinoembryonic antigen. 
Table II. Multivariate analysis of the clinicopathological factors related to responsiveness of therapy.

\begin{tabular}{lccccccr}
\hline & & & & & & \multicolumn{2}{c}{$95 \%$ CI for OR } \\
\cline { 5 - 8 } Variables & $\mathrm{B}$ & $\mathrm{SE}$ & Wals & P-value & OR & Lower & Upper \\
\hline Gender & -0.105 & 0.743 & 0.020 & 0.888 & 0.901 & 0.210 & 3.862 \\
Age (years) & 0.596 & 0.602 & 0.981 & 0.322 & 1.815 & 0.558 & 5.909 \\
Lymph node metastasis & -0.098 & 0.571 & 0.029 & 0.864 & 0.907 & 0.296 & 2.778 \\
Distant metastasis & 0.534 & 0.685 & 0.608 & 0.435 & 1.705 & 0.446 & 6.525 \\
Tumor location & -0.304 & 0.419 & 0.526 & 0.468 & 0.738 & 0.324 & 1.679 \\
Length & -0.186 & 0.350 & 0.283 & 0.595 & 0.830 & 0.418 & 1.648 \\
Tumor differentiation & 0.284 & 0.370 & 0.591 & 0.442 & 1.329 & 0.644 & 2.744 \\
Smoking history & 0.390 & 0.748 & 0.272 & 0.602 & 1.477 & 0.341 & 6.397 \\
Drinking history & -0.245 & 0.686 & 0.127 & 0.721 & 0.783 & 0.204 & 3.003 \\
Family history & -0.114 & 0.612 & 0.035 & 0.852 & 0.892 & 0.269 & 2.961 \\
Myelosuppression & 1.208 & 0.335 & 13.037 & 0.000 & 3.347 & 1.737 & 6.449 \\
miR-7 level & -1.330 & 0.565 & 5.541 & 0.019 & 0.265 & 0.087 & 0.800 \\
CEA & -1.287 & 0.564 & 5.214 & 0.022 & 0.276 & 0.091 & 0.833 \\
Cyfra21-1 & -1.216 & 0.585 & 4.314 & 0.038 & 0.296 & 0.094 & 0.934 \\
Constant & 4.092 & 2.421 & 2.858 & 0.091 & 59.877 & & \\
\hline
\end{tabular}

CEA, carcinoembryonic antigen; CI, confidence interval; OR, overall remission.
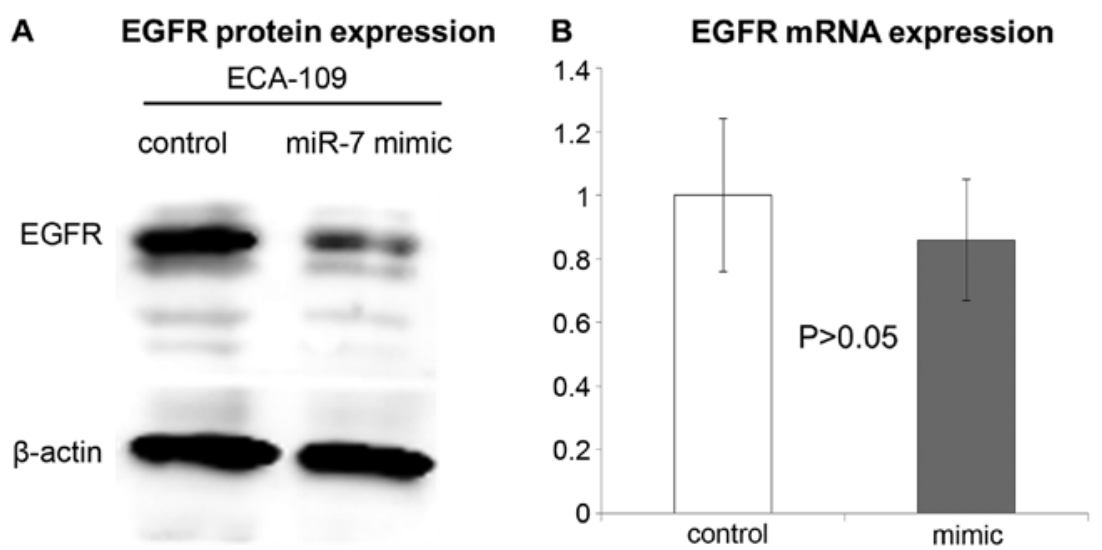

Figure 5. EGFR is a target of miR-7. miR-7 mimic transfection alters EGFR protein (A) expression but not mRNA levels (B) in ECA-109 cells.

Furthermore, the expression level analysis revealed that serum miR-7 is a valuable biomarker for differentiating the responsiveness to CRT of ESCC patients. As shown in Table II, the responsiveness to CRT is significantly associated with the levels of Cyfra21-1, CEA, miR-7 and myelosuppression before treatments. i.e. ESCC patients with lower Cyfra21-1 and CEA, higher miR-7 and severe myelosuppression were much more sensitive to CRT, which is similar to previous studies (45-47). However, this conclusion should be confirmed in a study with larger and more homogeneous samples.

The higher expression of miR-7 in ESCC may be more sensitive to CRT, which can be explained with activated EGFR and AKT associated pathways $(24,30)$. Recent studies have reported that EGFR can modulate DNA repair (48-50), consequently influence the responsiveness to CRT. While the present study showed that miR-7 has the ability to down- regulate the expression of EGFR, which is similar to other studies $(24,30,51)$. These results suggested that serum miR-7 may serve as a biomarker for the noninvasive diagnostic and predictive marker for ESCC.

In conclusion, the present study showed that serum miR-7 levels were significantly suppressed in patients with ESCC compared with control subjects, and the degree of suppression is correlated with longer tumor and positive lymph node metastasis. ESCC patients with lower Cyfra21-1 and CEA, higher miR-7, and severe myelosuppression were much more sensitive to CRT. These findings indicate that serum miR-7 may serve as a novel diagnostic and response predictive marker for ESCC patients, and it can also play a potential role in selecting CRT. Furthermore, we demonstrated that miR-7 mediated its function by repressing EGFR, which could be a novel mechanism of the ESCC patients responsiveness to CRT, suggesting that 
miR-7 possibly could be employed as an effective therapeutic target for ESCC.

\section{Acknowledgements}

We are grateful to Professor Cedric X. Yu (Department of Radiation Oncology, University of Maryland School of Medicine, Baltimore, MD, USA) for reviewing the manuscript.

\section{References}

1. Zhou ZG, Gao XS, Qiao XY and Zhang P: Literature analysis of radiotherapy for esophageal cancer in China. Chin J Cancer 29: 873-881, 2010.

2. Schneider BJ and Urba SG: Preoperative chemoradiation for the treatment of locoregional esophageal cancer: The standard of care? Semin Radiat Oncol 17: 45-52, 2007.

3. Shibata T, Kokubu A, Saito S, Narisawa-Saito M, Sasaki H, Aoyagi K, Yoshimatsu Y, Tachimori Y, Kushima R, Kiyono T, et al: NRF2 mutation confers malignant potential and resistance to chemoradiation therapy in advanced esophageal squamous cancer. Neoplasia 13: 864-873, 2011.

4. Fokas E, Weiss $C$ and Rödel C: The role of radiotherapy in the multimodal management of esophageal cancer. Dig Dis 31: 30-37, 2013.

5. Luthra R, Wu TT, Luthra MG, Izzo J, Lopez-Alvarez E, Zhang L, Bailey J, Lee JH, Bresalier R, Rashid A, et al: Gene expression profiling of localized esophageal carcinomas: Association with pathologic response to preoperative chemoradiation. J Clin Oncol 24: 259-267, 2006.

6. Wu X, Gu J, Wu TT, Swisher SG, Liao Z, Correa AM, Liu J, Etzel CJ, Amos CI, Huang M, et al: Genetic variations in radiation and chemotherapy drug action pathways predict clinical outcomes in esophageal cancer. J Clin Oncol 24: 3789-3798, 2006.

7. Zhang JX, Tong ZT, Yang L, Wang F, Chai HP, Zhang F, Xie MR, Zhang AL, Wu LM, Hong H, et al: PITX2: A promising predictive biomarker of patients' prognosis and chemoradioresistance in esophageal squamous cell carcinoma. Int J Cancer 132 2567-2577, 2013

8. Calin GA, Dumitru CD, Shimizu M, Bichi R, Zupo S, Noch E, Aldler H, Rattan S, Keating M, Rai K, et al: Frequent deletions and down-regulation of micro-RNA genes miR15 and miR16 at 13 q14 in chronic lymphocytic leukemia. Proc Natl Acad Sci USA 99: 15524-15529, 2002.

9. Liu Z, Jin ZY, Liu CH, Xie F, Lin XS and Huang Q: MicroRNA-21 regulates biological behavior by inducing EMT in human cholangiocarcinoma. Int J Clin Exp Pathol 8: 4684-4694, 2015.

10. Yang X, Ye J, Yan H, Tang Z, Shen 1, Zhang J and Yang L: MiR-491 attenuates cancer stem cells-like properties of hepatocellular carcinoma by inhibition of GIT-1/NF- $\kappa \mathrm{B}-$ mediated EMT. Tumour Biol: Jul 19, 2015 (Epub ahead of print).

11. Chang YL, Zhou PJ, Wei L, Li W, Ji Z, Fang YX and Gao WQ: MicroRNA-7 inhibits the stemness of prostate cancer stem-like cells and tumorigenesis by repressing KLF4/PI3K/Akt/p21 pathway. Oncotarget 6: 24017-24031, 2015.

12. Cui R, Meng W, Sun HL, Kim T, Ye Z, Fassan M, Jeon YJ, Li B, Vicentini C, Peng Y, et al: MicroRNA-224 promotes tumor progression in nonsmall cell lung cancer. Proc Natl Acad Sci USA 112: E4288-E4297, 2015.

13. Li W, Shen S, Wu S, Chen Z, Hu C and Yan R: Regulation of tumorigenesis and metastasis of hepatocellular carcinoma tumor endothelial cells by microRNA-3178 and underlying mechanism. Biochem Biophys Res Commun 464: 881-887, 2015.

14. Zhao L, Bode AM, Cao Y and Dong Z: Regulatory mechanisms and clinical perspectives of miRNA in tumor radiosensitivity. Carcinogenesis 33: 2220-2227, 2012.

15. Lu J, Getz G, Miska EA, Alvarez-Saavedra E, Lamb J, Peck D, Sweet-Cordero A, Ebert BL, Mak RH, Ferrando AA, et al: MicroRNA expression profiles classify human cancers. Nature 435: 834-838, 2005

16. Volinia S, Calin GA, Liu CG, Ambs S, Cimmino A, Petrocca F, Visone R, Iorio M, Roldo C, Ferracin M, et al: A microRNA expression signature of human solid tumors defines cancer gene targets. Proc Natl Acad Sci USA 103: 2257-2261, 2006.
17. Schwarzenbach H, Nishida N, Calin GA and Pantel K: Clinical relevance of circulating cell-free microRNAs in cancer. Nat Rev Clin Oncol 11: 145-156, 2014.

18. Mitchell PS, Parkin RK, Kroh EM, Fritz BR, Wyman SK, Pogosova-Agadjanyan EL, Peterson A, Noteboom J, O'Briant KC, Allen A, et al: Circulating microRNAs as stable blood-based markers for cancer detection. Proc Natl Acad Sci USA 105: 10513-10518, 2008.

19. Erson AE and Petty EM: MicroRNAs in development and disease. Clin Genet 74: 296-306, 2008.

20. Lee YS and Dutta A: MicroRNAs in cancer. Annu Rev Pathol 4: 199-227, 2009.

21. Kroh EM, Parkin RK, Mitchell PS and Tewari M: Analysis of circulating microRNA biomarkers in plasma and serum using quantitative reverse transcription-PCR (qRT-PCR). Methods 50: 298-301, 2010.

22. Webster RJ, Giles KM, Price KJ, Zhang PM, Mattick JS and Leedman PJ: Regulation of epidermal growth factor receptor signaling in human cancer cells by microRNA-7. J Biol Chem 284: 5731-5741, 2009.

23. Buckley AF, Burgart LJ, Sahai V and Kakar S: Epidermal growth factor receptor expression and gene copy number in conventional hepatocellular carcinoma. Am J Clin Pathol 129: 245-251, 2008 .

24. Kefas B, Godlewski J, Comeau L, Li Y, Abounader R, Hawkinson M, Lee J, Fine H, Chiocca EA, Lawler S, et al: microRNA-7 inhibits the epidermal growth factor receptor and the Akt pathway and is down-regulated in glioblastoma. Cancer Res 68: 3566-3572, 2008.

25. Reddy SD, Ohshiro K, Rayala SK and Kumar R: MicroRNA-7, a homeobox D10 target, inhibits p21-activated kinase 1 and regulates its functions. Cancer Res 68: 8195-8200, 2008.

26. Fang Y, Xue JL, Shen Q, Chen J and Tian L: MicroRNA-7 inhibits tumor growth and metastasis by targeting the phosphoinositide 3-kinase/Akt pathway in hepatocellular carcinoma. Hepatology 55: 1852-1862, 2012.

27. Zhang N, Li X, Wu CW, Dong Y, Cai M, Mok MT, Wang H, Chen J, Ng SS, Chen M, et al: microRNA-7 is a novel inhibitor of YY1 contributing to colorectal tumorigenesis. Oncogene 32: 5078-5088, 2013.

28. Li $\mathrm{X}$ and Carthew RW: A microRNA mediates EGF receptor signaling and promotes photoreceptor differentiation in the Drosophila eye. Cell 123: 1267-1277, 2005.

29. Pogribny IP, Filkowski JN, Tryndyak VP, Golubov A, Shpyleva SI and Kovalchuk O: Alterations of microRNAs and their targets are associated with acquired resistance of MCF-7 breast cancer cells to cisplatin. Int J Cancer 127: 1785-1794, 2010.

30. Lee KM, Choi EJ and Kim IA: microRNA-7 increases radiosensitivity of human cancer cells with activated EGFR-associated signaling. Radiother Oncol 101: 171-176, 2011.

31. Dong W, Li B, Wang Z, Zhang Z and Wang J: Clinical significance of microRNA-24 expression in esophageal squamous cell carcinoma. Neoplasma 62: 250-258, 2015.

32. Miller AB, Hoogstraten B, Staquet $M$ and Winkler A: Reporting results of cancer treatment. Cancer 47: 207-214, 1981.

33. Schmittgen TD, Lee EJ and Jiang J: High-throughput real-time PCR. Methods Mol Biol 429: 89-98, 2008.

34. Wieder HA, Brücher BL, Zimmermann F, Becker K, Lordick F, Beer A, Schwaiger M, Fink U, Siewert JR, Stein HJ, et al: Time course of tumor metabolic activity during chemoradiotherapy of esophageal squamous cell carcinoma and response to treatment. J Clin Oncol 22: 900-908, 2004.

35. Suzuki A, Xiao L, Hayashi Y, Macapinlac HA, Welsh J, Lin SH, Lee JH, Bhutani MS, Maru DM, Hofstetter WL, et al: Prognostic significance of baseline positron emission tomography and importance of clinical complete response in patients with esophageal or gastroesophageal junction cancer treated with definitive chemoradiotherapy. Cancer 117: 4823-4833, 2011.

36. Vaupel P and Harrison L: Tumor hypoxia: Causative factors, compensatory mechanisms, and cellular response. Oncologist 9 (Suppl 5): S4-S9, 2004.

37. Jamal M, Rath BH, Williams ES, Camphausen K and Tofilon PJ: Microenvironmental regulation of glioblastoma radioresponse. Clin Cancer Res 16: 6049-6059, 2010.

38. Thoms J and Bristow RG: DNA repair targeting and radiotherapy: A focus on the therapeutic ratio. Semin Radiat Oncol 20: 217-222, 2010. 
39. Langerak P and Russell P: Regulatory networks integrating cell cycle control with DNA damage checkpoints and double-strand break repair. Philos Trans R Soc Lond B Biol Sci 366: 3562-3571, 2011.

40. Chou $\mathrm{CH}$, Chen SU and Cheng JC: Radiation-induced interleukin-6 expression through MAPK/p38/NF-kappaB signaling pathway and the resultant antiapoptotic effect on endothelial cells through Mcl-1 expression with sIL6-Ralpha. Int J Radiat Oncol Biol Phys 75: 1553-1561, 2009.

41. Jiang L, Liu X, Chen Z, Jin Y, Heidbreder CE, Kolokythas A, Wang A, Dai Y and Zhou X: MicroRNA-7 targets IGF1R (insulin-like growth factor 1 receptor) in tongue squamous cell carcinoma cells. Biochem J 432: 199-205, 2010.

42. Kong X, Li G, Yuan Y, He Y, Wu X, Zhang W, Wu Z, Chen T, $\mathrm{Wu}$ W, Lobie PE, et al: MicroRNA-7 inhibits epithelial-tomesenchymal transition and metastasis of breast cancer cells via targeting FAK expression. PLoS One 7: e41523, 2012.

43. Liu X, Jiang L, Wang A, Yu J, Shi F and Zhou X: MicroRNA-138 suppresses invasion and promotes apoptosis in head and neck squamous cell carcinoma cell lines. Cancer Lett 286: 217-222, 2009.

44. Liu X, Yu J, Jiang L, Wang A, Shi F, Ye H and Zhou X: MicroRNA-222 regulates cell invasion by targeting matrix metalloproteinase 1 (MMP1) and manganese superoxide dismutase 2 (SOD2) in tongue squamous cell carcinoma cell lines. Cancer Genomics Proteomics 6: 131-139, 2009.

45. Kunisaki C, Imada T, Yamada R, Hatori S, Kinbara K, Watai K, Akiyama H, Nomura M, Matsuda G, Otsuka Y, et al: Prognostic factors after chemoradiotherapy for patients with inoperable esophageal squamous cell carcinoma. Hepatogastroenterology 53 366-371, 2006
46. Wakatsuki M, Suzuki Y, Nakamoto S, Ohno T, Ishikawa $\mathrm{H}$, Kiyohara H, Kiyozuka M, Shirai K, Nakayama Y and Nakano T: Clinical usefulness of CYFRA 21-1 for esophageal squamous cell carcinoma in radiation therapy. J Gastroenterol Hepatol 22: 715-719, 2007.

47. Yi Y, Li B, Wang Z, Sun H, Gong H and Zhang Z: CYFRA21-1 and CEA are useful markers for predicting the sensitivity to chemoradiotherapy of esophageal squamous cell carcinoma. Biomarkers 14: 480-485, 2009.

48. Liccardi G, Hartley JA and Hochhauser D: EGFR nuclear translocation modulates DNA repair following cisplatin and ionizing radiation treatment. Cancer Res 71: 1103-1114, 2011.

49. Szumiel I: Epidermal growth factor receptor and DNA double strand break repair: The cell's self-defence. Cell Signal 18: 1537-1548, 2006.

50. Kriegs M, Kasten-Pisula U, Rieckmann T, Holst K, Saker J, Dahm-Daphi J and Dikomey E: The epidermal growth factor receptor modulates DNA double-strand break repair by regulating non-homologous end-joining. DNA Repair 9: 889-897, 2010.

51. Kalinowski FC, Giles KM, Candy PA, Ali A, Ganda C, Epis MR, Webster RJ and Leedman PJ: Regulation of epidermal growth factor receptor signaling and erlotinib sensitivity in head and neck cancer cells by miR-7. PLoS One 7: e47067, 2012. 